\title{
Estructuración urbana del centro histórico de San Salvador una reflexión primera y general
}

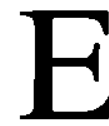

n la década de los ochentas, Salvatore -Toto- regresa a su pueblo después de treinta años de ausencia. Ha venido al funeral de su amigo Alfredo; el hombre que le enseño el manejo del proyector de peliculas de cine, algunas de las sutilezas del amor y a enfrentar la vida con valentía. El amigo más amigo de todos, el hombre quien, siendo el niño, salvó del incendio de la primera sala de cine del pueblo: la iglesia.

Después del siniestro, el más rico de aquel pueblo abriría la primera sala en forma: el cinema "Paradiso", donde Toto -acompañado y aconsejado por Alfredo ciego- proyectaría las escenas de besos más apasionados que harian vibrar a los expectadores gracias a la censura -por hecho y derecho- de la censura que hasta entonces habia ejercido el cura sobre las cintas. En "Paradiso" Toto conocería también el primero -y único-amor de su vida.

Cuando Toto regresa, todos están treinta años más viejos: él, su madre, el dueño del cinema, los expectadores y el loco, que treinta, cuarenta años atrás y después de cada función noctuma, los corría a todos de la plaza central reiterándoles ser el dueño absoluto de ella...

Alfredo amorosamente ha dejado su herencia para Salvatore: la edición de todas las escenas de besos, censuradas por el cura cuando el cine era la iglesia o la iglesia era también el cine... Pero entonces el cinema "Paradiso" está en ruinas, abandonado... Ya no es negocio, pues

- Antropologa mexicana, investigadora del Instituto Nacional de Antropología e Historia (INAH), Tabasco/México. Este trabajo fue elaborado en 1995, durante su estadía como profesora invitada en el Departamento de Arquitectura de la UCA. 
ahora la gente prefiere ver la tele y los videos... La municipalidad de la ciudad lo ha comprado para levantar en su terreno una magnifica cons. trucción moderna a tono con los tiempos. Así que después del funeral de Alfredo y estando Toto todavia en su pueblo, él y todos aquellos que compartieron las delicias del Paraíso, asisten con profunda pena, a la demolición, de una vez con dinamita; de aquel lugar, de aquel edificio. donde anidan tantos recuerdos colectivos e individuales. Afortunadamente, también en esta triste ocasión, el loco -enmedio de un mar de vehiculos automotores- sin correrlos esta vez, a todos les recuerda quién tiene la propiedad absoluta de la plaza...

\section{Una reflexión primera y general}

Los depositarios inmediatos del patrimonio cultural e histórico que constituyen los espacios e inmuebles de poblados y ciudades son, en primera instancia sus habitantes. Ellos y sus antepasados lo han formado y conformado. En ese patrimonio se ven a sí mismos como pueblo y como individuo; en él radica el testimonio tangible de muchos momentos de su pasado y su presente colectivo; en él se finca buena parte de su memoria histórica, y él constituye una de las bases de su identidad $y$ herencia.

Los centros historicos de las ciudades han llegado a considerarse patrimonio urbano, cultural, histórico y universal dentro de un arduo proceso de toma de conciencia durante el siglo XX, sobre lo que constituye la herencia de los pueblos del y para el mundo. A través de coloquios y reuniones de diferentes organismos europeos e internacionales, que se inician desde antes de la segunda guerra mundial', se han ido estableciendo los conceptos de los bienes tangibles que conforman el patrimonio histórico cultural entre ellos los centros urbanos e históricos ${ }^{2}$, las normas y recomendaciones para su identificación, protección, conservación y rehabilitación ${ }^{3}$. Y junto con el trabajo propiamente científico, en el cual se ha adentrado en el análisis de los aspectos y componentes del complejo "centro histórico"4 que apoyan los objetivos de protección del patrimonio cultural urbano, se han sentado las bases para implementar el marco jurídico de su defensa y declaratoria como patrimonio no solo de las naciones que los detentan en su suelo sino de toda la humanidad.

El concepto de "centro histórico" deviene de la compleja interrelación de elementos de orden social, económico, político, cultural y espacial no sólo de nuestro propio momento histórico sino de varios momentos que van desde el origen del asentamiento hasta la ciudad de nuestros días. Abordar y comprender esta compleja interrelación requiere del esfuerzo y voluntad de todas las instancias socio políticas y económicas que necesariamente se ven involucradas en la ciudad y que tienen intereses en ella y en este espacio singular; por lo mismo se 
requiere también del esfuerzo interdisciplinario e interinstitucional, as! como la voluntad para coordinar y continuar acciones efectivas en políticas educativas, de protección, conservación y rehabilitación.

Pero al intervenir en los centros históricos con acciones y planteamientos parciales y/o elitistas, olvidando que los destinatarios inmediatos de estas acciones son los mismos habitantes del centro histórico y de la ciudad, no se alcanzan a resolver problemas prioritarios y urgentes como son vivienda, servicios, transporte y seguridad, ni se cimienta un legado patrimonial tan singularmente rico en testimonios y vestigios de diferentes momentos históricos para las generaciones futuras.

Las páginas siguientes se ubican en una de las dos grandes vertientes en que se abordan los estudios y cimiento de acciones políticas, sociales, económicas y culturales sobre los centros históricos: el análisis de su origen y desarrollo como espacio urbano "centro histórico", es decir su estructuración. La otra vertiente es el análisis -para la intervención- de las condiciones socioeconómicas y culturales, históricas, incluyendo las de la historia reciente de su población.

En la primera vertiente donde ubicamos esta breve reseña de la conformación urbana del centro histórico de San Salvador, hay varios elementos más que no se han abordado aqui, pero que es importante mencionar por ser complementarios a ello: 1) la identificación y catalogación del "haber" inmobiliario (construcciones) de los centros históricos, las condiciones en las que se encuentran, el uso que se les da, su datación y tipología; 2) la peculiaridad formal, histórica espacial, de la trama urbana como elemento característico de un centro histórico determinado; 3) los elementos naturales (cuerpos de agua, lomas, quebradas -topografía-) que se han ido integrando en el tiempo al centro históricos. Estos tres elementos conforman, cada uno a su manera, también al espacio "centro histórico" y no sólo porque así lo aconsejen las recomendaciones internacionales, sino porque son testimonios históricos de la vida de la ciudad que, como elementos individuales, al igual que el espacio todo, son y deben ser suceptibles de protección y conservación jurídica y cultural.

Tanto al abordar el estudio de la estructuración del centro histórico de San Salvador como de cualquier otro centro, así como para llevar a cabo cualquier otra acción que lo contemple como un espacio particular, se presenta el primer problema teórico metodológico; su delimitación geográfica, hasta dónde y por qué hasla ahl. Para ello no basta identificar el haber arquitectónico —que ha sido la manera común para la delimitación de la mayorfa de los centros histórico latinoamericanos-, peor aún si lo "valioso" se considera únicamente a lo construido durante la época colonial o a lo que tiene características arquitectónicas conspicuas ${ }^{6}$. Es necesario identificar en la organización economico-social de diferentes momentos, aquellas relaciones sociales, políticas, económicas y culturales que tuvieron la fuerza de expresarse espacialmente y dejar su impronta en 
una determinada forma y organización urbana. Asimismo identificar en ellas el momento en que podemos delimitar, así, geográfica e históricamente el centro histórico. Para nuestro centro histórico este momento "geográfico" -asf como para muchas ciudades latinoamericanas que lo comparten- es el inicio del fenómeno de metropolización de las ciudades, que en San Salvador empieza a manifestarse hacia los años treintas de este siglo. En ese momento, la ciudad colonial, la del siglo XIX, con su traza y sus barrios pasan a constituirse en el "centro" de una ciudad de mayor dimensión y complejidad; el espacio urbano empieza a diferenciarse clasistamente con mayor profundidad y de manera horizontal; la urbanización, aunque de manera errática, ya tiene una dirección de distinta cualidad a la que tuvo durante los siglos anteriores.

Este es el principal objetivo que pretenden las páginas siguientes, al exponer someramente la conformación y los actores del centro histórico de San Salvador.

\section{El espacio de la ciudad colonial y sus estructuras fundamentales}

Pasadas dos décadas del año 25 del siglo XVI, el Ayuntamiento ya instalado en un lugar conocido hoy como "La Bermuda" en el valle del Suchitoto decidió, con la anuencia de la Real Audiciencia de Guatemala, trasladar la villa de San Salvador, centro de la Provincia del mismo nombre, al valle de "Las Hamacas", a los pies del volcán Quezaltepeque?. Un valle que aunque tal, es de suelo accidentado, con lomas y pendientes, cuya parte más baja está hacia el oriente. Lo circunscriben las quebradas del Tutunichapa al norte, la Mascota al surponiente, la de Monserrat al sur centro y el río Acelhuate al sur oriente. Por su altura respecto al río, así como sus lomas, está resguardado de inundaciones, pero no de los temibles movimientos telúricos, muchas veces acompañados de erupciones de lava, lapilli y cenizas ardientes del San Salvador, que marcaron desde entonces el sino de la ciudad hasta nuestros días. 
Fig. 1

\section{Croquis que muestra los rasgos topográficos generales del valle de Las Hamacas o de Quezaltepeque}

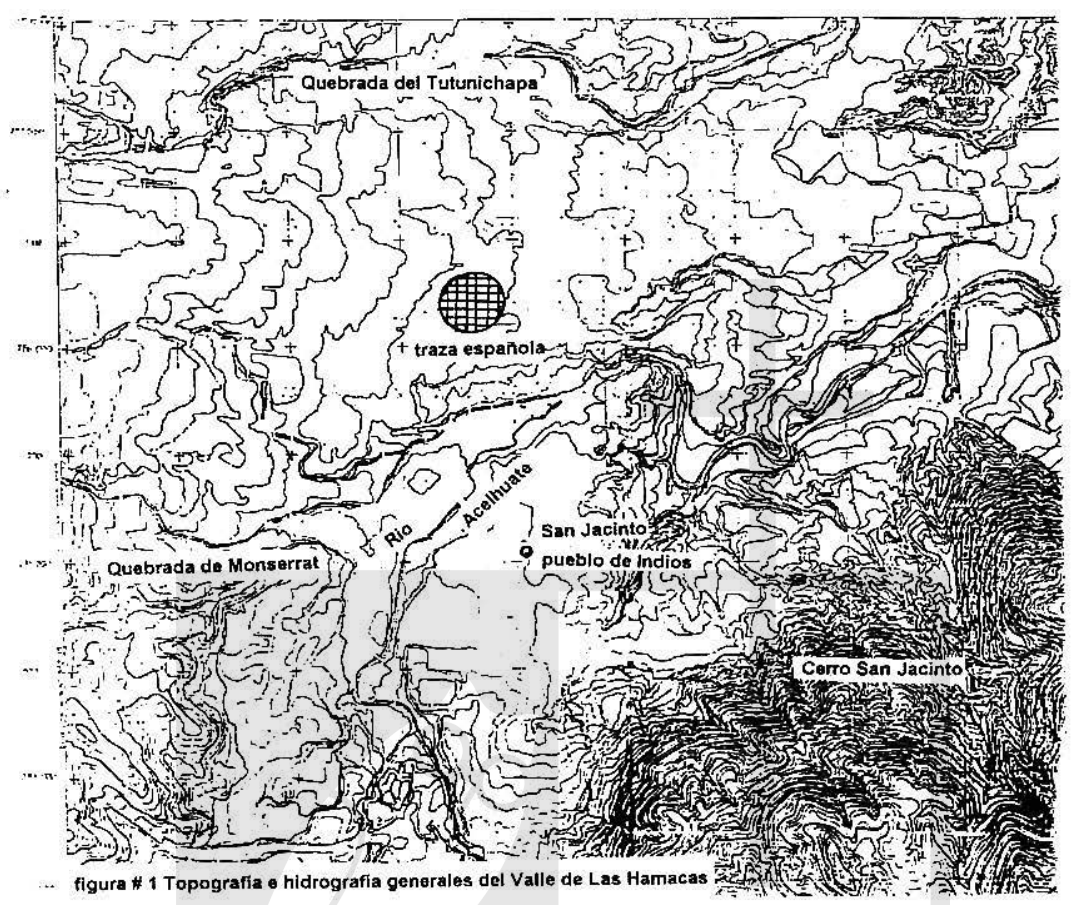

En la parte oriente del valle que baja hacia el río, el mismo Ayuntamiento de la "ciudad vieja", trazó la segunda San Salvador, a la manera que lo hicieron los españoles desde los primeros años de conquista: una plaza central de la cual partieran dos ejes, uno norte-sur y oriente-poniente con sus paralelas ${ }^{9}$, casi tres décadas antes que Felipe II ordenara en los años setentas, la regulación de las trazas en forma de damero, sus puntuales medidas y la ubicación de los edificios de importancia.

Allí dispusieron el lugar de la plaza (actual plaza "Libertad"), el de la parroquia, del cabildo, del Hospital y repartieron en propiedad los solares a los "vecinos", mayormente encomenderos. La más cercana fuente de abastecimiento de mano de obra y de materias primas para la construcción o actividad productiva fue el pequeño pueblo de indios de San Jacinto, emplazado al sur de que aquella traza, cruzando el Acelhuate.

Poco después de fundada, en 1546 a la nueva villa del valle de Las Hamacas le fue concedido el título de "ciudad", y en 1551 empezaron a llegar las órdenes 
religiosas regulares a quienes el Ayuntamiento fue asignándoles sus lugares, diferenciados de la traza española. Primero artibaron los frailes dominicos que instalaron su convento a orillas del río y que posteriormente trasladaron a su asiento definitivo (actual Catedral y probablemente también el parqueo universitario). Quizás hayan sido ellos también los fundadores de la ermita del Calvario (actual iglesia del Sagrado Corazón).

Pocos años después, la ermita de la Virgen de la presentación se fincó a la orilla norte de aquella traza española. Esta ermita se les atribuye a los franciscanos, quienes hasta los años setentas empezaron a construir su convento en ese mismo emplazamiento de la ermita, pero que posteriormente dejaron para construirlo a la orilla oriente (actual Mercado ex-cuartel y su manzana adyacente al oriente).

Después del segundo gran terremoto que sufrí esta nueva ciudad española en 1594, cuyas consecuencias fueron de "ruina completa", los mercedarios solicitaron solares para su convento, pero no fue sino hasta 1623 que empezaron a construirlo enmedio de una ciudad todavía devastada en la orilla suroriente de la traza, fincando asimismo hacia el oriente la emita de San Esteban.

La presencia y actuación de los frailes regulares fue el impulso para la creación y desarrollo de la otra estructura urbana constitutiva de la ciudad colonial: el barrio; pues a través de los conventos, iglesias y ermitas, asl como de la relación socioeconómica y cultural de las órdenes con la población, éstas y sus "cofradias", conformaron los núcleos de esas estructuras urbanas, diferenciadas de la traza española, tanto por su organización espacial como social, económica, política y cultural.

Es el desarrollo de estos barrios, lo que constituye durante la época colonial hasta principios del siglo XIX, las expansiones de la ciudad; pero no sólo, también el que la primera traza española se haya constituido en el CENTRO de aquella ciudad.

Dentro del proceso socio político y económico de gran parte de las colonias americanas, deben destacarse las Reformas Borbonicas hacia el último tercio del siglo XVIII, pues tuvieron una incidencia notable en la reorganización territorial y urbana. Particularmente para nuestra ciudad, significaron el control de ésta sobre la producción agrícola y ganadera, así como su comercialización, principalmente de los productos "estancados" por la Corona como el tabaco y el añil. Espacialmente ésto se expresó en la instalación de las garitas de control de las Rentas del Tabaco y las Alcabalas, con las cuales la ciudad definió por primera vez sus fronteras y las de algunos de sus barrios, a más del desarrollo de la infraestructura político-económica dentro de la antigua traza española. 
Fig. 2

\section{Croquis que muestra la ubicación de las garitas}

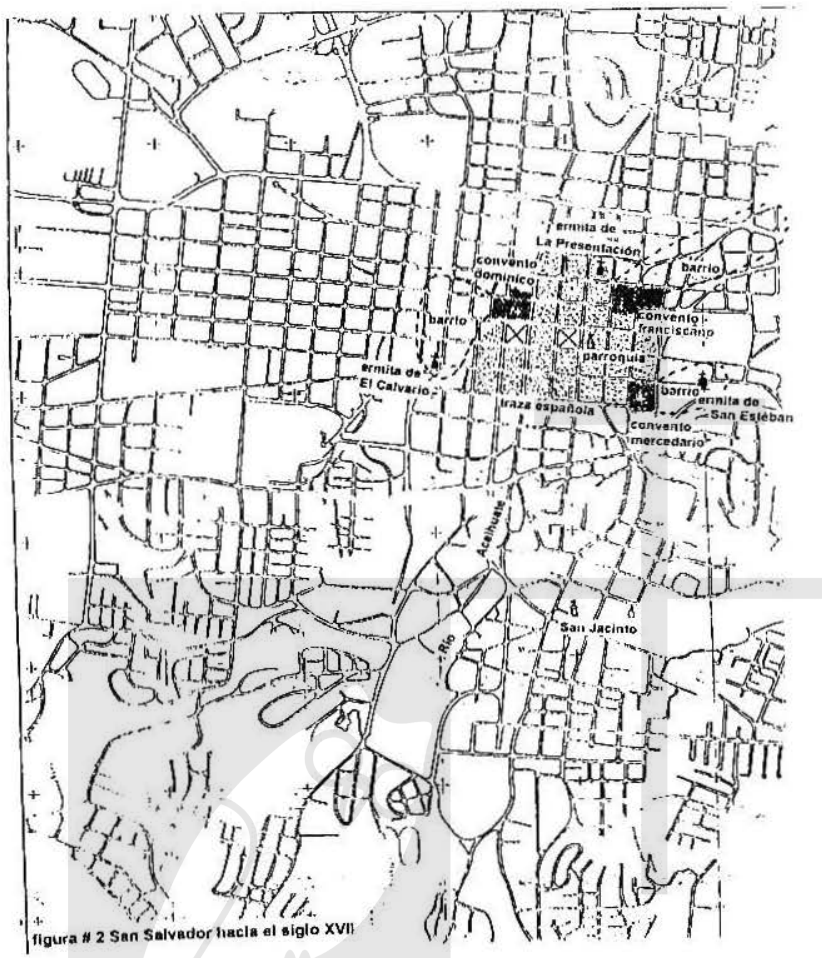

En esta visión somera de la conformación espacial de la ciudad durante la época colonial, debemos señalar un factor de importancia: después de fundada la segunda San Salvador, ésta sufrió durante el siglo XVI dos terremotos, uno de ellos de consecuencias devastadoras. Asimismo, en el siglo XVII, dos más, también terribles, asolaron a la ciudad y contribuyeron a dificultar enormemente la reconstrucción que se trataba de implementar desde 1594. En el siglo XVIII, la ciudad fue objeto de otro sísmo, aunque de menor envergadura que los anteriores. Con todo ello cabe preguntarse ¿cómo puede afirmarse un hecho espacial como la permanencia de la original traza española? Para afirmar la permanencia de este hecho nos apoyamos en dos cuestiones, la primera son las fuentes históricas que señalan que aún cuando las edificaciones importantes, tales como las iglesias y conventos, cabildo, hospital y plazas se vieron seriamente afectadas e incluso los sísmos llegaron a destruir algunas de ellas, fueron siempre reedificadas en el mismo lugar. La segunda se basa en la observación de las manzanas centrales. Aquí salta a la vista su peculiaridad en forma, tamaño y alineación, com- 
paradas con manzanas que corresponde a expansiones posteriores. Esto nos permite considerar como muy factible que aún cuando la traza española original haya sido destruída por los terremotos, su trazo original sirví́ siempre de guía a trazos posteriores.

\section{Dos momentos de la ciudad del siglo XIX}

Puede decirse que durante las primeras siete décadas del siglo XIX, el espacio urbano de San Salvador, es, con una pequeña expansión hacia sur, poniente y norte, el mismo de la ciudad de los últimos años coloniales, aún cuando se haya iniciado un nuevo proceso histórico de gran envergadura en lo social, politico y económico, que tiene como una de sus primeras consecuencias más relevantes hacia mediados del siglo - 1854-, la fundación de una nueva capital del pars: la Nueva San Salvador, de efímera primacía territorial, y expresión espacial de la necesidad política de un grupo económico emergente que empezada a fincar su riqueza en la producción cafetalera, desplazando para siempre la base económica colonial que hasta entonces habla sido el añil.

Una de las razones principales que explican este fenómeno espacial de relativa permanencia de la ciudad colonial de San Salvador es, precisamente, que esos años significaron la reorganización de la formación sociopolítica y económica que desembocó finalmente en la constitución del Estado Nacional. A partir de ese momento - la década de los años setentas del XIX - el espacio urbano empieza a ser reorganizado y a expresar el nuevo estado de cosas.

Como elemento constante en toda la historia urbana sansalvadoreña, los terremotos aportaron su parte en el siglo XIX. Dos temblores de tierra terribles uno en 1854 y el otro en 1879- contribuyeron a situaciones trascendentes para la ciudad. El primero fue el pretexto para fundar la Nueva San Salvador y mudar la cabeza política de la nación, así como empezar a modificar los usos de suelo y con ello la simbologla urbana colonial de algunas estructuras edilicias como fue el caso del convento franciscano, convertido a cuartel militar. El segundo, contribuyó al derribamiento definitivo de varias de aquellas construcciones, la remodelación de otras con nuevos estilos arquitectónicos, la construcción de la infraestructura propia del Estado y de una sociedad pujante que requerfa de una infraestructura "cultural" de élite, y a las primeras modificaciones de la trama urbana colonial, dividiéndose antiguas manzanas y trazándose nuevas calle a manera de paseos arbolados y agradables como la avenida "Independencia".

Asimismo fue necesario empezar a implementar una infraestructura urbana para la movilización del Capital emanado de la producción de cafe, que entre otras cosas, en estos años, dio lugar al tendido de la vía férrea entre Santa Tecla (Nueva San Salvador) y San Salvador y la construcción de la terminal ferrocarrilera en la ciudad. 
Hacia los años noventas la infraestructura urbana de San Salvador estaba bien y bastante acondicionada para ello: bancos, bodegas, hoteles, oficinas, etc. los barrios coloniales empezaron a expandirse y el antiguo pueblo de indios de San Jacinto dejo de serlo para vincularse a la ciudad como uno más de sus barrios. Los propietarios del suelo empezaron a considerar rentable la posible urbanización de tierras agrícolas, adyacentes a la capital.

Figura 3

\section{Croquis de la ciudad de San Salvador entre 1870 y 1890}

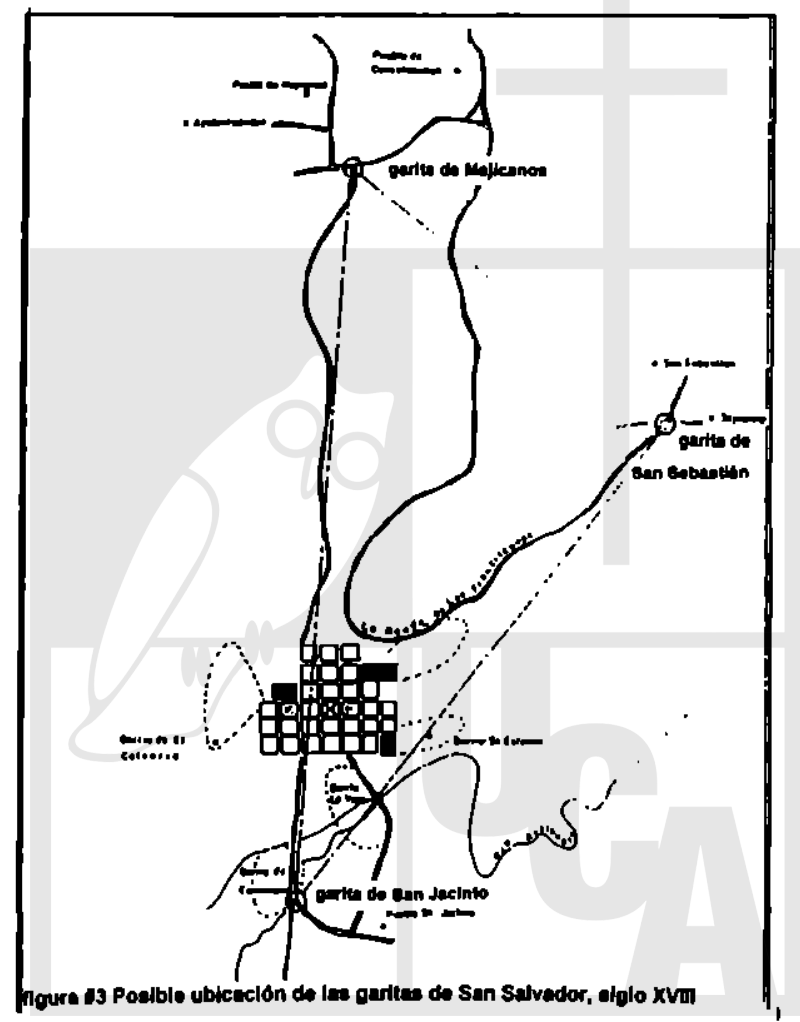

La contraparte de este proceso enunciado fue que la producción cafetalera requirió de la expropiación de tierras pertenecientes a las comunidades indias, con lo cual se inició el proceso de liberación de la fuerza de trabajo agrícola y los primeros movimientos migratorios a las ciudades de San Salvador y Santa Ana, principalmente. 
La boyante producción cafetalera a finales del siglo XIX, dio lugar al impulso de la manufactura que en la ciudad empezó a desarrollarse con la producción de loza, cerveza, jabón, telas y zapatos. Se instalaron fábricas en las afueras de la ciudad y talleres en el "centro" (nuestra vieja traza española) y los barrios. Las primeras grandes casas comerciales extranjeras empezaron a operar, también en ese "centro".

\section{La delimitante histórico geográfica del centro histórico}

De esta manera, el siglo XX encuentra al país y a su ciudad capital, inmersos en el proceso de desarrollo del sistema capitalista de producción; empezando a diferenciarse con claridad, el Capital del Trabajo; con un Estado Nacional constituido y la incipiente formación de una clase obrera; asimismo con la presencia definitoria en el desarrollo económico-político del Capital inglés, todo lo cual se ha comenzado a expresar en la organización del espacio urbano, en su ampliación y diversificación.

La ciudad ha iniciado su expansión hacia el poniente, sobre las tierras altas del valle de las Hamacas, sobre dos ejes principales: las actuales calles Rubén Darío y Manuel Arce. La población trabajadora, inmigrante y oriunda de la ciudad, se asienta en los antiguos barrios coloniales y sus afueras donde se han instalado las fábricas y los talleres, y se ha iniciado un fenómeno socio arquitectónico nuevo: el mesón.

Figura 4

Croquis que muestra el espacio de la ciudad a principios del siglo $\mathrm{XX}$

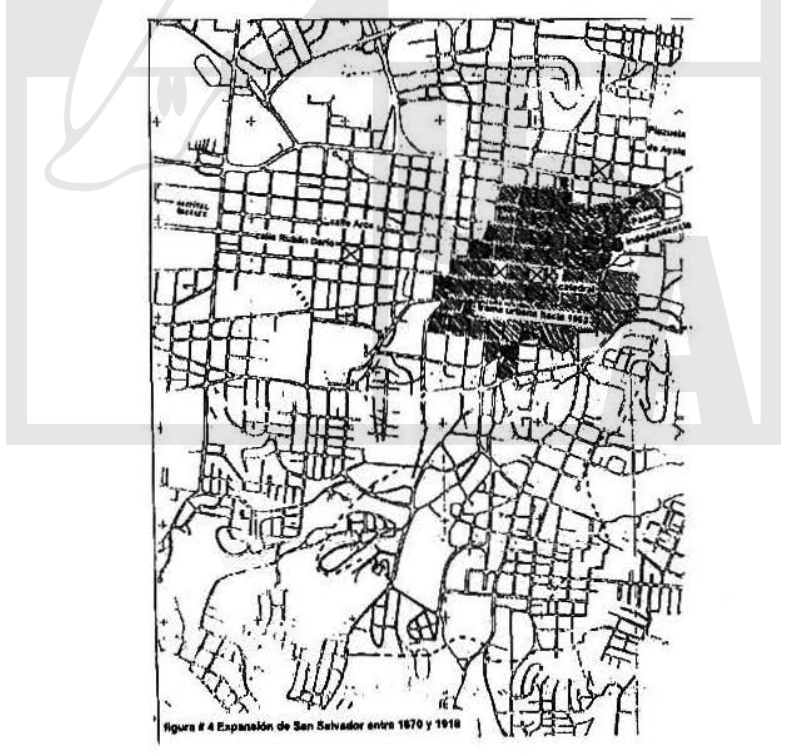


Es entre 1918 y 1929 , es decir, entre finales de la primera guerra mundial y la primera gran crisis del capitalismo en el siglo XX con sus años de depresión, que ese proceso iniciado hacia los años setentas del siglo XIX empieza a consolidarse, para finalmente en la década de los treintas quedarlo definitivamente. El capital norteamericano ha desplazado al inglés y ha empezado a imponer y definir la política socioeconómica en Centroamérica; las organizaciones de trabajadores han avanzado al grado de estar en la posibilidad de constituir el primer partido clasista de El Salvador, el Partido Comunista.

Fue en este período en que la ciudad todavía con su impronta colonial, experimenta su primera gran expansión del siglo $\mathrm{XX}$, que implica la división horizontal clasista del suelo urbano: las clases propietarias abandonan su vivienda en el "centro" y los barrios de la ciudad - sin renunciar a la propiedad del suelo ni de las edificaciones- para trasladarse hacia el poniente del valle. Nacen nuevos barrios y "colonias" obreras hacia el norte y oriente de la ciudad; todo ello "pensando" de una nueva manera: la urbanización definida y dirigida ya por las clases propietarias de las antiguas fincas agrícolas, sobre todo productoras de café, ya por las instancias gubernamentales.

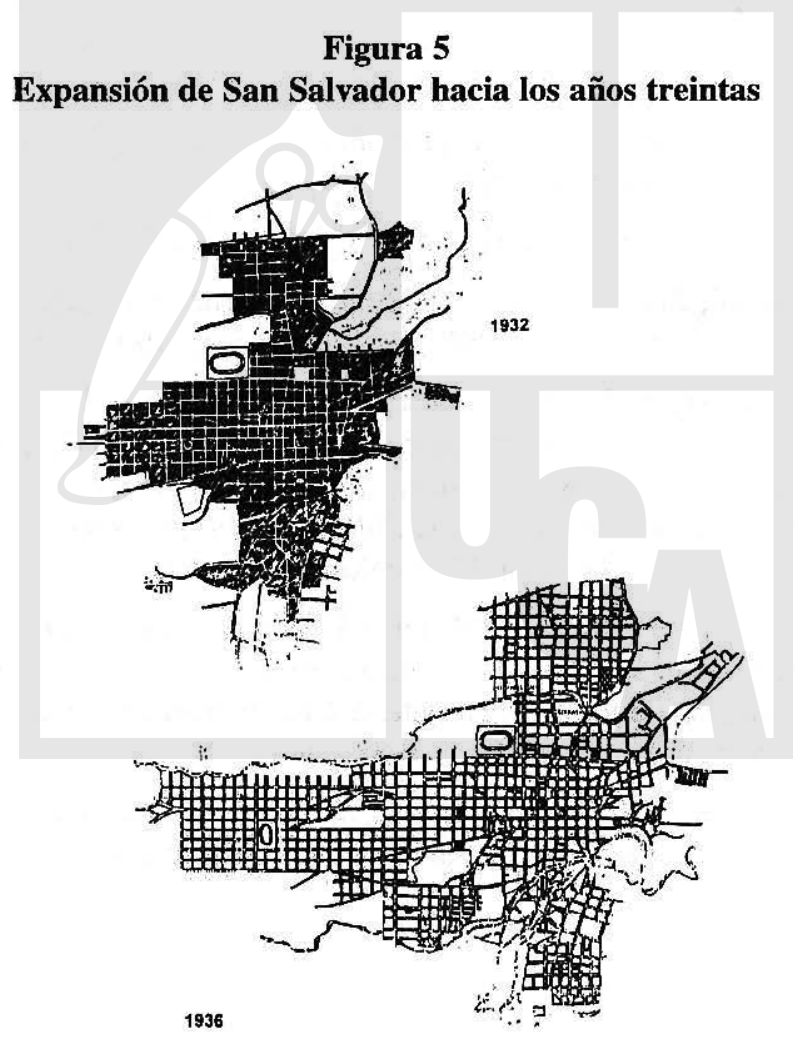


Este período de tan singular importancia marca historicamente la aparición de un nuevo "centro" urbano, ahora constituido por aquel espacio que ocupaba la ciudad colonial: su traza y sus barrios. Al mismo tiempo delimita históricamente su geograffa, pues se diferencia en su proceso de formación y desarrollo de un nuevo fenómeno urbano que está por comenzar: la metropolitización de San Salvador. Las antiguas edificaciones que habitaban las clases propietarias empiezan a subdividirse, para convertirse también en "mesones". Este espacio viejo quedó entonces para la habitación y trabajo de las clases trabajadoras, dándole de esta manera la connotación popular que hasta la fecha conserva.

Si consideramos que los "centros históricos" tienen, entre otras características, la de ser los testimonio espaciales de diferentes momentos de la historia de una ciudad, el "centro histórico" de San Salvador se constituye históricogeograficamente por esas estructuras espaciales de su momento colonial (traza y barrios), por las expansiones y modificaciones sufridas durante sus dos momentos importantes del siglo XIX, y por los primeros espacios de expansión del siglo XX hasta los años treintas, que marcan, éstos últimos, un momento de diferenciación cualitativa en el proceso de urbanización de nuestra ciudad.

\section{Bibliografia}

Barón Castro, Rodolfo La población de El Salvador, UCA Editores, 2' ed., San Salvador, Centroamérica, 1978.

Daltón Roque El Salvador, Monogralía UCA Editores, 5² ed. El Salvador, 1994.

Manuel Mármol, Los sucesos de 1932 en El Salvador. Colecc. Testigos de la Historia, vol. 7, 1' ed., UCA Editores, El Salvador, 1993.

García, Miguel Angel. Diccionario histórico enciclopédico de la república de El Salvador desde la conquista hasto el año de 1894 en lo político, social, ciencias, letras y bellas artes, homenaje en su CD centenario de haber sido elevada a la categoría de ciudad 1546-1946. Primer tomo, imprenta Nacional república de EI Salvador, Centro América, 1952.

Lungo, Mario y Sonia Baires. Población y economía en la consolidación de la capital salvadoreña: 1880-1930 en: La estructuración de las capitales centroamericanas, Editorial Universitaria Centroamericana, Colecc. Rueda del Tiempo, San José de Costa Rica, 1988.

Salazar L. Flora. Estructuras urbanas. El barrio de (la) concepción de San Salvador, 1553-1926, en El Salvador en construcción, julio de 1996, San Salvador. 


\section{Notas}

1. El primer antecedente, particularmente del patrimonio urbano se halla en la Carta del Urbanismo, Atenas 1933. En ella se establecen los principios rectores para la organización de las ciudades (europeas), que permitieran remediar su "situación actual" (después de la primera guerra mundial) Domingo García Ramos, citado por Salvador Díaz-Berrio F. en Protección del patrimonio cultural urbano, Colecc. Fuentes, Instituto Nacional de Antropología e Historia INAH-, México, 1986 (en adelante Díaz B).

2. A partir del Convenio Italiano de Gubbio (1960), se dan por primera vez los elementos básicos con respecto a la rehabilitación relativa a los centros urbanos e históricos y que posteriormente, sobre todo después de 1972, se irían precisando. Sin embargo mucho antes, incluso en el siglo XIX, se dieron proyectos aislados en Europa, los EUA y México que tenían como objetivo a conjuntos urbanos. El concepto "centro histórico" se menciona como tal, ya con todo su acervo y riqueza de elementos constitutivos en Quito, Ecuador.

3. Es en la Convención de la UNESCO (1972), donde se establecen los pasos básicos y lógicos para las acciones de intervención sobre el patrimonio cultural (Díaz B, op cit).

4. Los pioneros en el estudio de los centros hitóricos latinoamericanos son Jorge Enrique Hardoy y Mario Dos Santos. Asimismo la Convención de la UNESCO, el Coloquio de ICOMOS (también en 1972), y la Carta de Venecia (1964), enmarcan el desarrollo de estos estudios científicos y de otros realizados para ciudades latinoamericanas como Quito y Guatemala. (Consúltese a Díaz B, op cit., y a José Antonio Teran Bonilla en Metodología de Investigación de Centros Históricos, Facultad de Arquitectura, División de Estudios de Posgrado, 1 ed., México, 1990.

5. Es en la Convención de la UNESCO mencionada que se toma en cuenta al patrimonio natural como un elemento complementario y necesario del patrimonio cultural y lo que su desaparición implica para el patrimonio de todos los pueblos del mundo. (Díaz B, opc. cit.).

6. La Carta Internacional de Venecia considera y de los lineamientos para salvaguardar las obras modestas de arquitectura que "con el tiempo han adquirido valor cultural". Es decir la arquitectura popular o vernacula. El valor patrimonial de esta arquitectura es uno de los elementos que se ha considero como importantes en el patrimonio inmobiliario de los centros históricos y forman parte de su acervo de elementos conformadores, (consultese a Díaz B, op. cit.).

7. Los españoles le dieron el nombre de volcán de San Salvador, y popularmente se le ha conocido como "El Jabalí" por su singular y característica forma.

8. Ciudad Vieja" es el nombre con el que hoy se le conoce a esta primera fundación de San Salvador, correspondiente a la fase de la conquista de América. En ese momento tenía la categoría de "villa" no de "ciudad".

9. En realidad la orientación de la ciudad no es norte sur, sino noreste suroeste. 